\title{
A Formalism for the Analysis and Design of Time and Path Diversity Schemes in Wireless Sensor Networks
}

\author{
Martin Haenggi \\ University of Notre Dame, IN 46556, USA \\ mhaenggi@nd.edu
}

\begin{abstract}
For Rayleigh fading channels, there exists an interesting similarity between resistive circuits and time and path diversity mechanisms in multihop wireless sensor networks. A resistor-like circuit element, the erristor, representing the normalized noise-to-signal ratio, is introduced. Given an end-to-end packet delivery probability (as a QoS requirement), the nonlinear mapping from link reception probabilities to erristor values greatly simplifies the problems of power allocation and the selection of time and path diversity schemes. Thanks to its simplicity, the formalism that is developed also provides valuable insight into the benefits of diversity mechanisms, which is illustrated by a number of examples.
\end{abstract}

\section{Introduction}

The lifetime of wireless sensor network is crucial, since autonomous operation must be guaranteed over an extended period [1,2]. Energy and interference considerations often necessitate multihop routing, where sensor nodes also act as routers, forwarding other nodes' packets [3]. Routing schemes that were developed for wired networks will perform suboptimally since they are based on virtually error-free point-to-point links, thereby ignoring two fundamental properties of the wireless link: 1) the fragility of the channel due to fading and interference $[4,5]$ and 2) the inherent broadcast property of wireless transmissions ${ }^{1}$. Whereas the first property is adverse, the second one can be exploited by transmission schemes that are based on the principle of cooperative diversity [6], where nodes coordinate both direct and relayed transmissions. Cooperative diversity is a form of spatial diversity, which, in the case of static singleantenna nodes, reduces to path diversity. The other promising strategy (in the case of narrowband channels) against fading is time diversity, which, for slow fading channels and relatively short packets, is mainly exploited in the form of retransmissions.

\footnotetext{
${ }^{1}$ We assume that omnidirectional antennas are employed.
} 
In this paper, we present a simple but powerful formalism that allows an efficient analysis and design of time and path diversity strategies for Rayleigh fading channels. In the analysis, the transmit power levels are given and the end-to-end reliability $p_{\mathrm{EE}}$ is to be determined, whereas in the (more interesting) design problem, we assume that the application dictates a certain end-to-end reliability $p_{\mathrm{D}}$, and the question is how to choose the transmit powers, the relays (paths), and the number of transmissions over each link in order to minimize energy consumption and/or maximize network lifetime under the constraint $p_{\mathrm{EE}} \geqslant p_{\mathrm{D}}$.

Example 1. Consider the simple example in Fig. 1. A packet is transmitted with reception probability $p_{01}$ over the first hop and transmitted twice over the second hop, with probabilities $p_{12,1}$ and $p_{12,2}$, respectively. The end-to-end reliability of the connection is $p_{\mathrm{EE}}=p_{01} \cdot\left(1-\left(1-p_{12,1}\right)\left(1-p_{12,2}\right)\right)$. Let $p_{\mathrm{D}}=90 \%$. What combination(s) of transmit

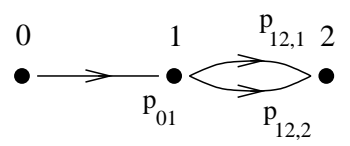

Fig. 1: A two-hop connection with two transmissions over the second hop. powers satisfy $p_{\mathrm{EE}} \geqslant p_{\mathrm{D}}$, and which one is energyoptimal?

\section{The Link Model}

We assume a narrowband multipath wireless channel, modeled as a slow Rayleigh fading channel [7] with an additive noise process $z$. The received signal at time $k$ is $y_{k}=a_{k} x_{k}+z_{k}$, where $a_{k}$ is the large-scale path loss multiplied by the fading coefficient. The variance of the noise process is denoted by $\sigma_{Z}^{2}$.

The transmission from node $i$ to node $j$ is successful if the signal-tonoise-and-interference ratio (SINR) $\gamma$ is above a certain threshold $\Theta$ that is determined by the communication hardware and the modulation and coding scheme [5]. With the assumptions above, $\gamma$ is a discrete random process with exponential distribution $p_{\gamma}(x)=1 / \bar{\gamma} e^{-x / \bar{\gamma}}$ with mean

$$
\bar{\gamma}=\frac{\bar{P}}{\sigma_{Z}^{2}+\sigma_{I}^{2}} .
$$

$\bar{P}$ denotes the average received signal power over a distance $d=\left\|x_{i}-x_{j}\right\|_{2}$ : $\bar{P}=P_{0} d^{-\alpha}$, where $P_{0}$ is proportional to the transmit power ${ }^{2}$, and the

\footnotetext{
2 This equation does not hold for very small distances. So, a more accurate model would be $\bar{P}=P_{0}^{\prime} \cdot\left(d / d_{0}\right)^{-\alpha}$, valid for $d \geqslant d_{0}$, with $P_{0}^{\prime}$ as the average value at the reference point $d_{0}$, which should be in the far field of the transmit antenna. At $916 \mathrm{MHz}$, for example, the near field may extend up to $3-4 \mathrm{ft}$ (several wavelengths).
} 
path loss exponent is $2 \leqslant \alpha \leqslant 5 . \sigma_{I}^{2}$ is the interference power affecting the transmission. It is the sum of the received power of all the undesired transmitters.

The following theorem shows that for Rayleigh fading networks, it is possible to analyze noise and interference separately.

Theorem 1. In a Rayleigh fading network, the reception probability $\mathbb{P}[\gamma \geqslant$ $\Theta$ ] can be factorized into the reception probability of a zero-noise network and the reception probability of a zero-interference network.

Proof: The probability that the SINR is bigger than a given threshold $\Theta$ follows from the cumulative distribution $f_{\gamma}(x)=1-e^{-x / \bar{\gamma}}$ :

$$
\begin{aligned}
\mathbb{P}[\gamma \geqslant \Theta] & =e^{-\Theta / \bar{\gamma}}=e^{-\frac{\Theta}{P}\left(\sigma_{Z}^{2}+\sigma_{I}^{2}\right)} \\
& =e^{-\frac{\Theta \sigma_{Z}^{2}}{P}} \cdot e^{-\frac{\Theta \sigma_{I}^{2}}{P}}=\mathbb{P}\left[\gamma_{Z} \geqslant \Theta\right] \cdot \mathbb{P}\left[\gamma_{I} \geqslant \Theta\right],
\end{aligned}
$$

where $\gamma_{Z}:=P / \sigma_{Z}^{2}$ denotes the signal-to-noise ratio (SNR) and $\gamma_{I}:=$ $P / \sigma_{I}^{2}$ denotes the signal-to-interference ratio (SIR). The first factor is the reception probability in a zero-interference network as it depends only on the noise, and the second factor is the reception probability in a zero-noise network, as it depends only on the interference. It follows from (2) that $\bar{\gamma}=\left(\bar{\gamma}_{Z} \bar{\gamma}_{I}\right) /\left(\bar{\gamma}_{Z}+\bar{\gamma}_{I}\right)$.

This allows an independent analysis of the effect caused by noise and the effect caused by interference. The focus of this paper is put on the noise, i.e., on the first factor in (2). If the load is light (low interference probability), then SIR $\gg \mathrm{SNR}$, and the noise analysis alone provides accurate results. For high load, a separate interference analysis ${ }^{3}$ has to be carried out, as in [9]. Most energy-constrained sensor networks aim at minimizing the communication, which justifies the focus on noise.

In a zero-interference network, the reception probability over a link of distance $d$ at a transmit power $P_{0}$, is given by

$$
p_{r}:=\mathbb{P}\left[\gamma_{Z} \geqslant \Theta\right]=e^{-\frac{\Theta \sigma_{Z}^{2}}{P_{0} d^{-\alpha}}} .
$$

Solving for $P_{0}$, we get for the necessary transmit power to achieve $p_{r}$ :

$$
P_{0}=\frac{d^{\alpha} \Theta \sigma_{Z}^{2}}{-\ln p_{r}}
$$

\footnotetext{
${ }^{3}$ Note that power scaling, i.e., scaling the transmit powers of all the nodes by the same factor, does not change the SIR, but (slightly) increases the SINR. This has been pointed out also in [8]
} 


\section{The Erristor Representation}

\subsection{Connections without retransmission}

Assume a $n$-hop connection from node 0 to node $n$ in a wireless sensor network. The desired end-to-end reliability is $p_{\mathrm{D}}$. The reception probability over a chain of $n$ nodes is

$$
p_{\mathrm{EE}}=e^{-\Theta \sum_{i=1}^{n} \frac{1}{\bar{\gamma}_{i}}}
$$

where $\bar{\gamma}_{i}$ denotes the mean SNR at receiver $i$. Let $R$ denote the normalized average noise-to-signal ratio (NSR) at the receiver, i.e., $R:=\Theta / \bar{\gamma}$. We get

$$
-\ln p_{\mathrm{EE}}=\sum_{i=1}^{n} R_{i}=R_{\mathrm{tot}} .
$$

Hence the condition $p_{\mathrm{EE}} \geqslant p_{\mathrm{D}}$ translates into the condition that the sum or the series connection ${ }^{4}$ of the NSR values $R_{i}$ is at most $R_{\mathrm{D}}:=-\ln p_{\mathrm{D}}$. So, the individual $R_{i}$ 's can be replaced by an equivalent $R_{\text {tot }}$. For a single link, we have

$$
R=-\ln p_{r} \quad \Longleftrightarrow \quad p_{r}=e^{-R} .
$$

For probabilities close to 1 ( or $R \ll 1$ ), the following first-order approximations are accurate:

$$
\hat{R}:=1-p_{r} \lesssim R \quad \Longleftrightarrow \quad \hat{p}_{r}:=1-R \lesssim p_{r}
$$

This approximation shows that for small values, the NSR can be considered equivalent to the packet error probability. To emphasize this fact and the resistor-like series connection property of the NSR, we denote $R$ as an "erristor" and its value as its "erristance".

Eq. (8) shows that, when determining the erristance from a given probability, the approximated value will be on the safe side, and from (6), we see that over a multihop connection, the noise accumulates and the error probabilities simply add up.

The relationship between the transmit power and $R$ is

$$
P_{0}=d^{\alpha} \bar{\gamma} \sigma_{Z}^{2}=\frac{d^{\alpha} \Theta \sigma_{Z}^{2}}{R} .
$$

Henceforth, $P:=d^{\alpha} / R$ denotes the normalized (by $\Theta \sigma_{Z}^{2}$ ) transmit power.

\footnotetext{
${ }^{4}$ In terms of the SNR values, this corresponds to a parallel connection, which was pointed out in [10].
} 
Example 2. Fig. 2 (left) shows an example with three links and their reception probabilities. From (6) we know that a series of hops translates into a series connection of erristors, hence we find the corresponding erristor network in Fig. 2 (right). For $p_{\mathrm{D}}=90 \%$, for example, the value of $R_{1}+R_{2}+R_{3}$ must be at most $-\ln p_{\mathrm{D}} \approx 0.105$. If all the power levels are equal, this can be achieved by setting $R_{1}=R_{2}=R_{3}=0.105 / 3=0.035$. A possible solution with unequal power is $R_{1}=R_{2}=0.05$ and $R_{3}=0.005$. Here, the probability after two links is $e^{-0.05} e^{-0.05} \approx 90.5 \%$, which is already close to $90 \%$. Consequently, a lot of of energy is consumed at the third link to ensure packet reception with the required probability $e^{-0.005}=99.5 \%$.
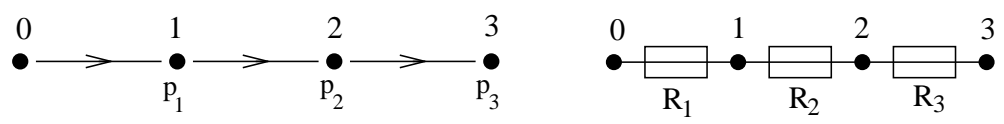

Fig. 2: A three-hop connection with link reception probabilities $p_{1}, p_{2}$, and $p_{3}$ (left) and the erristor circuit (right). The erristor values are the normalized noise-to-signal ratios $R_{1}, R_{2}$, and $R_{3} . R_{i}=-\ln p_{i}$.

If the internode distances $d_{i}$ (between node $i-1$ and node $i$ ) are given, a solution can be determined that ensures that all the transmit power levels have the same value $P$. From $\left(d_{1}^{\alpha}+d_{2}^{\alpha}+d_{3}^{\alpha}\right) / P \leqslant-\ln p_{\mathrm{D}}$, we get

$$
P \geqslant \frac{d_{1}^{\alpha}+d_{2}^{\alpha}+d_{3}^{\alpha}}{-\ln p_{\mathrm{D}}}
$$

For $d_{i}=i, \alpha=2$, and $p_{\mathrm{D}}=90 \%$, for example, we get $P \approx 14 \cdot 9.5 \approx 133$ and $R_{1} \approx 0.0075, R_{2} \approx 0.03$, and $R_{3} \approx 0.0677$.

\subsection{Connections with time diversity (retransmissions)}

Coming back to example 1 (Fig. 1), the question is how to incorporate retransmissions into the erristor formalism. Considering the second link, we found that $p_{12}=1-\left(1-p_{12,1}\right)\left(1-p_{12,2}\right)$, which is equivalent to

$$
p_{12}=1-\left(1-e^{-R_{12,1}}\right)\left(1-e^{-R_{12,2}}\right) .
$$

In general, for $n$ transmissions over one link at NSR levels $R_{i}$, we have

$$
p_{n}=1-\prod_{i=1}^{n}\left(1-e^{-R_{i}}\right) .
$$

To derive a general rule for the simplification of these expressions, we apply the following theorem. 


\section{Theorem 2.}

$$
\text { For } \begin{aligned}
\left(x_{1}, x_{2}, \ldots, x_{n}\right) \in & \left(\mathbb{R}_{0}^{+}\right)^{n}, \\
& 1-\prod_{i=1}^{n}\left(1-e^{-x_{i}}\right) \geqslant e^{-\prod_{i=1}^{n} x_{i}} .
\end{aligned}
$$

The identity holds if and only if $\prod_{i=1}^{n} x_{i}=0$.

The proof is presented in the Appendix.

Example 1 (cont.). So, in example $1, e^{-R_{12,1} R_{12,2}}$ is a lower bound for $p_{12}$, and for $R_{1} \ll 1$ and $R_{2} \ll 1$, the bound is tight. Thus we may replace the erristors $R_{21}$ and $R_{22}$ by an erristor $R_{2}=R_{21} R_{22}$. In the erristor diagram, the two transmissions are illustrated by a parallel connection (see Fig. 3). So,

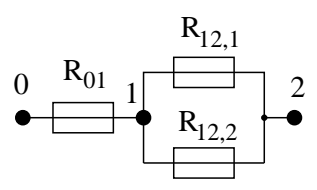

Fig. 3: The erristor circuit of Fig. 1. erristors connected in series behave like regular resistors, whereas the values of erristors connected in parallel have to be multiplied. Due to the bound derived above, the resultant end-to-end reliability will be slightly higher than the one required.

For $n$ transmissions with the same power level $R$, the difference between the precise probability value $1-\left(1-e^{-R}\right)^{n}$ and the lower bound $e^{-R^{n}}$ is plotted in Fig. 4. The erristance threshold where the bound is within $1 \%$ is $R=0.236$ for $n=2$ and $R=0.375$ for $n=4$. Thus for $R<1 / 4(p>78 \%)$, the bound is sufficiently tight for all practical purposes.

For values $R \gtrsim 1$, the bound is loose, and the multiplication does not make sense, since the overall erristance increases, although, of course, even a retransmission with low power still leads to an improvement in the total link reception probability. However, for $R \gtrsim 1 / 2$, a single transmission outperforms splitting the power into two transmissions: For two transmissions at NSR $2 R$, the reception probability is $p_{r}=1-\left(1-e^{-2 R}\right)^{2}$, whereas for a single transmission at NSR $R$, we get $p_{r}^{\prime}=e^{-R}$. The two probabilities are equal for

$$
R=\ln 2-\ln (\sqrt{5}-1) \approx 0.48 .
$$

So, for $R \gtrsim 1 / 2$, the reception probability is higher for a single transmission at NSR $R$.

Note that a peak transmit power constraint $P<P_{\max }$ translates into the minimum resistor value that can be used. Over a link of distance $d$, the minimum erristor value is $R_{\min }=d^{\alpha} / P_{\max }$. 


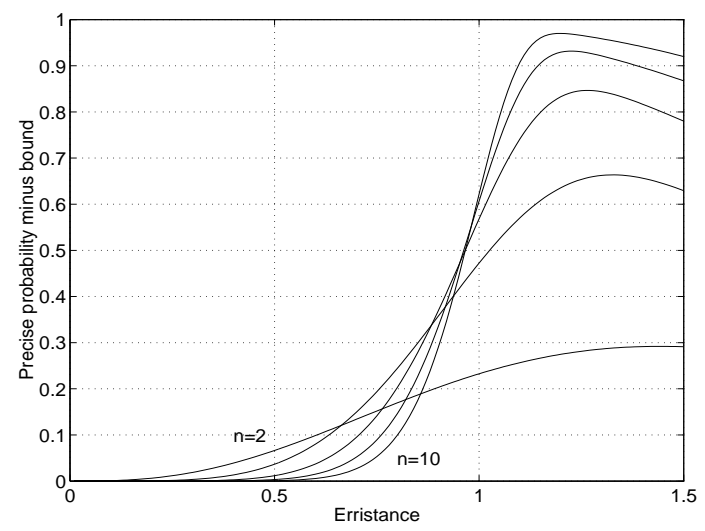

Fig. 4: The difference between the exact probability and the lower bound for $n=$ $2,4,6,8,10$ transmissions with equal erristance $R$.

\section{Path Diversity}

\subsection{Transmissions over independent paths}

The analysis in the previous Section is valid if retransmissions have independent reception probabilities. This is not guaranteed if the channel's coherence time is substantially larger than a packet transmission time or if shadowing is the cause for packet loss, in which case a form of path diversity is required.

Example 3. Fig. 5 displays an example of a network where path independence is guaranteed even when the channels have a long coherence time. By conventional analysis, the end-to-end reception probability is

$$
\begin{aligned}
p_{\mathrm{EE}}= & \left(1-\left(1-e^{-R_{01}} e^{-R_{12}}\right)\left(1-e^{-R_{02}}\right)\right) \times \\
& \left(1-\left(1-e^{-R_{23}} e^{-R_{34}}\right)\left(1-e^{-R_{24}}\right)\right) .
\end{aligned}
$$

How to choose the $R_{i j}$ 's to guarantee $p_{\mathrm{EE}} \geqslant p_{\mathrm{D}}=95 \%$ ? This is a nontrivial question that can easily be answered using the erristor formalism. The equivalent erristor (see Fig. 5 (right)) is

$$
R_{\mathrm{tot}}=\left(R_{01}+R_{12}\right) R_{02}+\left(R_{23}+R_{34}\right) R_{24},
$$

and $p_{\mathrm{EE}}=e^{-R_{\mathrm{tot}}}$. For a desired $p_{\mathrm{D}}=95 \%$, we have $R_{\mathrm{tot}} \approx 0.05$. Thanks to the symmetry, $\left(R_{01}+R_{12}\right) R_{02}=\left(R_{23}+R_{34}\right) R_{24}=0.025$ is a solution; hence we may set nearest-neighbor hops to $R_{i-1, i}=0.05$ and $R_{02}=R_{24}=0.25$. Note that the value for the two longer hops is 5 times 

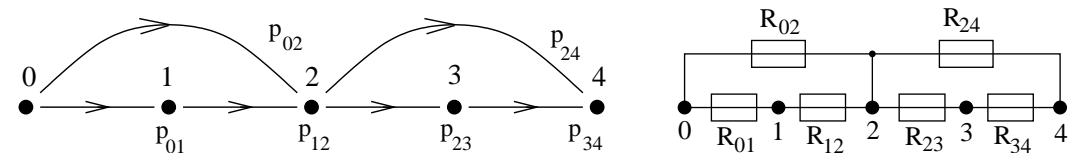

Fig. 5: A network that exploits path diversity (left) and its erristor circuit (right).

bigger, which means that the necessary transmit powers are comparable if the nodes have equal distances and the path loss exponent is between 2 and 3 . So, using the erristor formalism, the diversity scheme and power allocation should guarantee $p_{\mathrm{EE}}=e^{-0.05} \approx 95.1 \%>p_{\mathrm{D}}$. The conventional analysis (15) yields $p_{\mathrm{EE}} \approx 95.8 \%$ which is, as expected, slightly bigger than the one from the erristor analysis. The formalism also permits a rapid reallocation of resources, if necessary. Assume node 3 runs out of energy. With $R_{34} \rightarrow \infty$, we see immediately that $R_{23}$ becomes useless, and the only path in the lower half of the diagram will be the one with $R_{24}$. What value does $R_{24}$ need to have to ensure $p_{\mathrm{D}}$ ? Without changing the other erristances, we immediately find $R_{24}=0.025$.

The total energy consumption (per packet) at each node can easily be determined:

$$
E_{i}=\sum_{j=1}^{m} \frac{d_{i j}^{\alpha}}{R_{i j}},
$$

where $m$ is the number of outgoing paths from node $i$.

\subsection{Implicit transmissions}

In the first example (Fig. 1), if node 2 listens to the transmission from node 0 to node 1 , then this implicit transmission has to be modeled by an additional erristor for an accurate analysis. This implicit erristor is free in terms of transmit power (but still requires power to receive the packet $)^{5}$.

Assume $p_{\mathrm{D}}=99 \%$, so $R_{\mathrm{tot}} \approx 0.01$. This is achieved by setting $R_{01}=$ 0.005 and $R_{12,1}=R_{12,2}=0.07$. However, since there is an implicit transmission from 0 to 2 , there is a erristor in parallel with a value of $R_{02}^{i}=$ $R_{01}\left(d_{02} / d_{01}\right)^{\alpha}$ (the superscript $i$ indicates an implicit transmission). Assuming $d_{02}=2 d_{01}$ and $\alpha=3$, we get $R_{02}^{i}=0.04, R_{\text {tot }}=0.01 \cdot 0.04$ and $p_{\mathrm{EE}} \approx 99.96 \%$, which is much better than the target of $99 \%$. So we can

\footnotetext{
${ }^{5}$ This advantage of omnidirectional transmission is often denoted as the wireless multicast advantage $[5,11]$.
} 
reduce $R_{01}$ to a value that guarantees $\left(R_{01}+0.005\right) R_{01} \cdot 88=0.01$. Solving the resulting quadratic equation yields $R_{01} \approx 1 / 30$, which corresponds to less than $1 / 6$ of the original power.

For large path loss exponents and smaller transmit powers, the benefit to listeners that are farther away than the intended receiver becomes small, since the implicit erristances will be close to one or even above However, if the implicit receiver is closer than the intended one or if the transmit power is relatively high, it is worthwile having the nodes awake and listening.

Example 3 (cont.). In example 3 (Fig. 5), there is an implicit transmission from node 2 to node 3 when node 2 is transmitting to node 4 . If node 3 ignores this transmission, then the analysis in the previous Subsection was correct. If it takes advantage of that information, we have to add another erristor, as shown in Fig. 6.

Assuming equal distances between neighboring nodes, $R_{01}^{i}=2^{-\alpha} R_{02}$ and

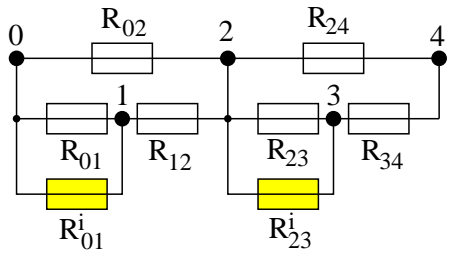

Fig. 6: The erristor circuit for Fig. 5 including implicit transmissions. Implicit erristors are grayshaded.

$R_{23}^{i}=2^{-\alpha} R_{24}$. For $\alpha=2$ and using the same values as before, $R_{i, i-1}=0.05$ and $R_{02}=R_{24}=0.25$, we find $R_{01}^{i}=R_{23}^{i}=0.0625$ and

$$
R_{\mathrm{tot}}=2\left(\frac{1}{20} \cdot \frac{1}{16}+\frac{1}{20}\right) \cdot \frac{1}{4} \approx 0.027,
$$

resulting in $p_{\mathrm{EE}} \approx 97.3 \%$, which is larger than the target of $95 \%$. Considering that $1 / 16 \ll 1$, we may try to omit the explicit transmission completely, which results in $R_{\text {tot }}=(1 / 16+1 / 20) / 2 \approx 0.056$ and $p_{\mathrm{EE}}=94.4 \%$. A slight decrease of $R_{02}$ and $R_{24}$ by $10 \%$ each brings $p_{\text {EE }}$ to $95.2 \%$.

In general, we can say that whenever there are two erristors in series with one significantly smaller than the other one, the power is better distributed differently.

Example 4. A simple cooperative scheme. In Fig. 7, a situation is shown where node 0 wants to transmit to node 1 , and the cooperative node $\mathrm{C}$ may help as a relay. From the erristor circuit it can be seen that there is no explicit transmission from 0 to $\mathrm{C}$. The goal is to determine which transmission strategy minimizes the total transmit energy $E_{\text {tot }}$ given a certain total erristance $R_{\text {tot }}$.

Let $D:=\left(\frac{d}{2}\right)^{\alpha}$. With $R_{\text {tot }}=R_{01}\left(R_{0 C}^{i}+R_{C 1}\right)$ and $R_{0 C}^{i}=D R_{01}$, we get $R_{\mathrm{tot}}=R_{01}\left(R_{01} D+R_{C 1}\right)$ and $E_{\mathrm{tot}}=2^{\alpha} R_{01}+d^{\alpha} R_{C 1}=2^{\alpha}\left(R_{01}+D R_{C 1}\right)$. 

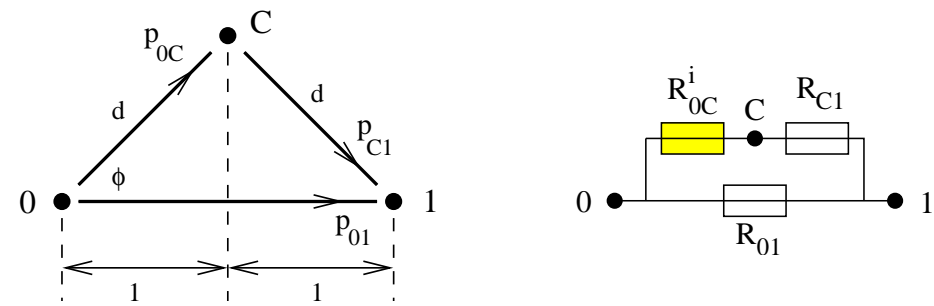

Fig. 7: A simple cooperative scheme (left) and the corresponding erristor circuit (right). Node $\mathrm{C}$ is relaying a packet from node 0 to assist node 0 . The distances $\overline{0 C}$ and $\overline{C 1}$ are $d=1 / \cos \phi$.

Strategy A: Equal received power. A possible strategy is to have $C$ transmit at a power level that makes the received power at node 1 equal to that from the direct transmission 01, i.e., $R_{C 1}=R_{01}=: R . R_{\text {tot }}$ simplifies to $R_{\text {tot }}=R^{2}(1+D)$, and thus $R=\sqrt{\frac{R_{\text {tot }}}{1+D}}$. Inserting this expression into the transmit energy $E_{\text {tot }}=\frac{2^{\alpha}}{R}(1+D)$ yields

$$
E_{\mathrm{tot}}^{A}=\frac{2^{\alpha}(1+D)^{3 / 2}}{\sqrt{R_{\mathrm{tot}}}}
$$

Strategy B: Equal transmit power. Here, we assume that both node 0 and $\mathrm{C}$ use the same transmit power. With $R:=R_{01}$ and $R_{C 1}=R_{0 C}^{i}=$ $R D$ we have $R_{\text {tot }}=2 D R^{2}$ and $R=\sqrt{\frac{R_{\text {tot }}}{2 D}}$. The total energy consumption is simply $E_{\mathrm{tot}}=2 \cdot 2^{\alpha} / R$, or, as a function of $R_{\mathrm{tot}}$,

$$
E_{\mathrm{tot}}^{B}=2^{\alpha+1} \sqrt{\frac{2 D}{R_{\mathrm{tot}}}} .
$$

The energy consumption ratio of strategies A and B is

$$
\rho:=\frac{E_{\mathrm{tot}}^{B}}{E_{\mathrm{tot}}^{A}}=\frac{2 \sqrt{2 D}}{(1+D)^{3 / 2}} .
$$

$\rho=1$ for $D=1$ and $D=\sqrt{5}-2 \approx 0.236$. For $\sqrt{5}-2<D<1$, strategy A is preferable $(\rho>1)$. The maximum $\rho$, however, is only $\frac{4}{9} \sqrt{6} \approx 1.089$, occurring at $D=\frac{1}{2}$. So, strategy $\mathrm{A}$ is at most $8.9 \%$ better.

To get a complete view, we also discuss the case of a direct one-hop transmission and an explicit two-hop scheme without a direct path from 0 to 1 . For the one-hop case, we have $E_{\text {tot }}^{\text {one }}=2^{\alpha} / R_{\text {tot }}$, and for the two hop case (assuming equal transmit powers), there are two erristors in series with value $R_{\text {tot }} / 2$ and thus $E_{\text {tot }}^{\text {two }}=4 d^{\alpha} / R_{\text {tot }}=4 D E_{\text {tot }}^{\text {one }}$. 
The one-hop strategy is better if $4 D \geqslant 1$, or, in terms of the actual distance $d, d>2^{1-2 / \alpha}$. So, for $\alpha=2$ and for $d \geqslant 2$, one-hop is always better, even for $\alpha \rightarrow \infty$, which is easily explained, since node $C$ is then not closer than node 1 . As a function of the angle $\phi=\arccos (1 / d)$, the condition for one-hop to be better is expressed as $\phi \geqslant \arccos \left(2^{2 / \alpha-1}\right)$. For $\alpha \rightarrow \infty$, the critical angle is $\phi=\pi / 3$ (corresponding to an equilateral triangle $0 \mathrm{C} 1$ ), as expected.

The last step is the comparison of these simple schemes with the cooperative strategies $\mathrm{A}$ and B. First we note that B always outperforms the two-hop scheme, since it exploits "free" information that is transmitted over the direct path. The tournament between A and onehop is won by A if $R_{\text {tot }}<(1+D)^{-3}$, and B wins against one-hop if $R_{\text {tot }}<\frac{8}{D}$. The resulting division of the $\left(D, R_{\text {tot }}^{-1}\right)$-plane in the different strategies is shown in Fig. 8. The erristor formalism transforms complex logarithmic relationships into simple polynomial ones, which permits the analytical derivation of these boundaries.

Example 5. Virtual antenna arrays. Several nodes that are close may cooperate and act as a virtual antenna array, exploiting spatial diversity. The performance of such arrays was analyzed in [12] from an informationtheoretic perspective. Here, we are using the erristor formalism to compare these schemes with conventional multihop routing. Fig. 9 shows the erristor diagram of a simple scenario with two nodes assisting each other at the source, in the middle, and at the destination. So, instead of individual nodes, we have clusters of two nodes at positions 0,1 , and 2 . It is assumed that the intracluster distances are much smaller than the intercluster distances $d / 2$. When the source node in cluster 0 is transmitting to cluster 1 , his peer will receive that packet with probability (almost) one since $R_{00}^{i} \ll R_{01}$. In the next time slot, this peer node will transmit the same packet to cluster 0 . Hence, the same packet is delivered over four 
different paths. Similarly, cluster 1 relays the packet to cluster 2 over four paths. In the case that the actual destination node itself in cluster 2 does not correctly receive the packet, an additional short intracluster transmission is required, whose energy is neglected in the following analysis.
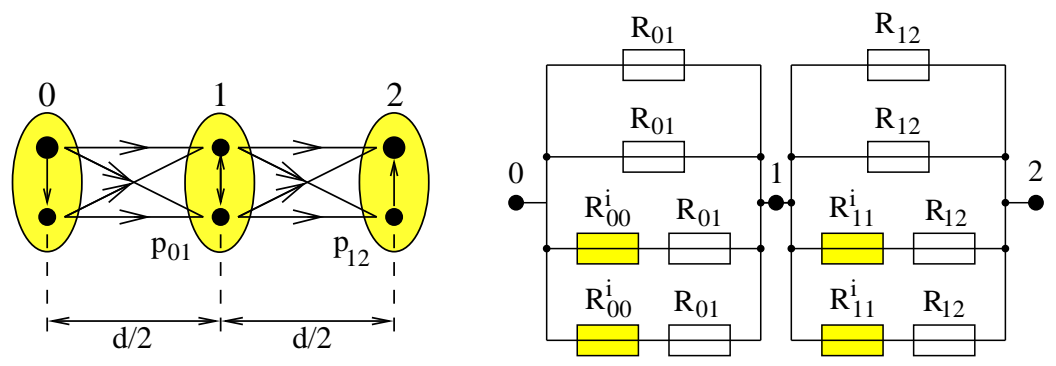

Fig. 9: A virtual antenna scheme and its erristor circuit.

We assume $R_{01}=R_{12}:=R$. For the diversity scheme, with $R_{00}^{i} \ll R$, we get $R_{\mathrm{tot}}=2 R^{4}$ and

$$
E_{\mathrm{tot}}=4 \frac{(d / 2)^{\alpha}}{R}=4\left(\frac{d}{2}\right)^{\alpha}\left(\frac{2}{R_{\mathrm{tot}}}\right)^{\frac{1}{4}}
$$

For comparison, for a 4-hop connection with hops of length $d / 4$, we have $R_{\mathrm{tot}}=4 R^{\prime}$ and

$$
E_{\mathrm{tot}}^{\prime}=4\left(\frac{d}{4}\right)^{\alpha} \frac{4}{R_{\mathrm{tot}}}=2^{4-\alpha}\left(\frac{d}{2}\right)^{\alpha} R_{\mathrm{tot}}^{-1}
$$

The ratio between the two energies is

$$
\frac{E_{\text {tot }}}{E_{\text {tot }}^{\prime}}=R_{\text {tot }}^{3} 2^{4 \alpha-7}
$$

Hence the diversity scheme is more efficient for

$$
R_{\text {tot }}<2^{\frac{4 \alpha-7}{3}} \quad \text { or } \quad p_{\mathrm{D}}>\exp \left(-2^{\frac{4 \alpha-7}{3}}\right) .
$$

This curve is plotted in Fig. 10 (left). Substantial energy gains are possible for high $p_{\mathrm{D}}$ (see Fig. 10 (right)). When the path loss exponent increases by one, the energy gain decreases by a factor of $2^{4 / 3} \approx 4 \mathrm{~dB}$. 

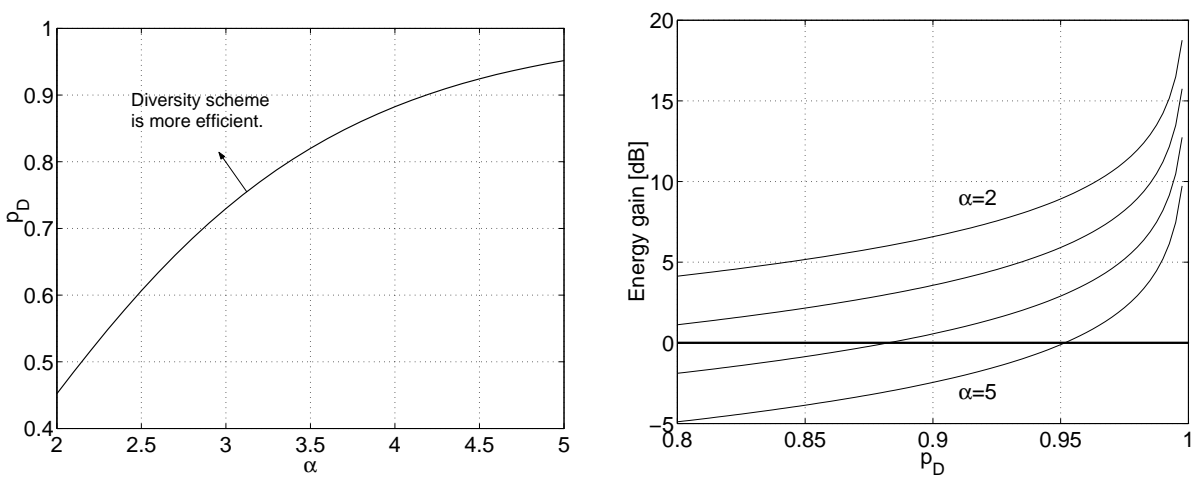

Fig. 10: Left: The region in the $\left(\alpha, p_{\mathrm{D}}\right)$ plane where the diversity scheme outperforms conventional multihop routing. Right: The energy gain as a function of the end-to-end probability.

This diversity scheme can be generalized to clusters of size $m$ that transmit over $n$ hops. In this case, $R_{\text {tot }}=n R^{m^{2}}$ and

$$
E_{\mathrm{tot}}=m n\left(\frac{d}{n}\right)^{\alpha}\left(\frac{n}{R_{\mathrm{tot}}}\right)^{\frac{1}{m^{2}}}
$$

For the multihop scheme with $m n$ hops $^{6}, R_{\text {tot }}=m n R^{\prime}$ and

$$
E_{\mathrm{tot}}^{\prime}=m n\left(\frac{d}{m n}\right)^{\alpha} \frac{m n}{R_{\mathrm{tot}}}
$$

The ratio is

$$
\frac{E_{\mathrm{tot}}}{E_{\mathrm{tot}}^{\prime}}=R_{\mathrm{tot}}^{1-\frac{1}{m^{2}}} n^{\frac{1}{m^{2}}-1} m^{\alpha-1}
$$

from which we see that the energy gain is maximized for $m=2$ (except for $\alpha=2$, where $m=3$ performs slightly better) and increases almost linearly in $n$. We conclude that for high $p_{\mathrm{D}}$ and smaller $\alpha$, the diversity scheme clearly outperforms conventional multihop routing.

\section{Concluding Remarks}

The erristor formalism permits the mapping of unhandy probability expressions into a simple circuit-like framework, which greatly simplifies the analysis and design of transmit schemes that are based on time diversity,

\footnotetext{
${ }^{6}$ This comparison is fair both in terms of the number of nodes involved and in the delay, since the total number of transmissions is $m n$ for both schemes.
} 
path diversity, or a combination thereof. The erristor circuit is topologically equivalent to the network graph and can therefore be drawn in a straightforward manner. Resource allocation and reallocation problems can effortlessly be solved by simple arithmetic, which makes a real-time implementation readily feasible. Further, the formalism may prove useful for educational purposes, since the multiplication property of parallel erristors impressively demonstrates the benefits of diversity schemes, and the series connection shows how the noise and, in turn, the error probability accumulates over multiple hops.

While this work is mainly focused on noise-limited networks, it seems possible to include the interference in the same framework, since SNR and SIR exhibit a parallel combination property similar to the SNR values of subsequent links (see Theorem 1). This will be part of future investigations.

\section{Appendix: Proof of Theorem 2}

From the inequality (see (13))

$$
1-\prod_{i=1}^{n}\left(1-e^{-x_{i}}\right) \geqslant e^{-\prod_{i=1}^{n} x_{i}}
$$

it is easy to see that both expressions are equal (to 1 ) if one of the $x_{i}$ is zero. It remains to show that $f(\cdot): \mathbb{R}^{n} \rightarrow \mathbb{R}$

$$
f_{n}\left(x_{1}, x_{2}, \ldots, x_{n}\right)=1-\prod_{i=1}^{n}\left(1-e^{-x_{i}}\right)-e^{-\prod_{i=1}^{n} x_{i}} .
$$

is positive if all $x_{i}$ are positive. We note that $f$ goes to zero if $\forall i, x_{i} \rightarrow \infty$. Hence $f$ is positive for positive $x_{i}$ if its partial derivatives $\partial f / \partial x_{i}$ are positive at 0 and have at most one zero for positive $x_{i}$. Since the function is symmetric in all $x_{i}$, it is sufficient to consider only one partial derivative. An inductive technique is employed, discussing the case $n=2$ first.

Consider

$$
g_{2}(x):=f_{2}(x, y)=e^{-x}+e^{-y}-e^{-x-y}-e^{-x y}
$$

for a fixed $y \geqslant 0$. For $y=0, g_{2}(x) \equiv 0$, and for $y>0$, we note that $g(0)=0$ and $\lim _{x \rightarrow \infty} g(x)=e^{-y}>0$. Since

$$
g^{\prime}(x)=\frac{d g}{d x}=e^{-x}\left(-1+e^{-y}+y e^{x(1-y)}\right),
$$


there exists a single local extremum $\bar{x}$ for $y>0$ at

$$
\bar{x}=\frac{1}{y-1} \ln \left(\frac{y}{1-e^{-y}}\right) .
$$

As $y>\left(1-e^{-y}\right)$ for $y>0$, we find $\bar{x}>0$ for $y>1$ and $\bar{x}<0$ for $0<y<1$. For $y=1$, no solution exists, and for $y=0, g^{\prime}(x) \equiv 0$. Since $g^{\prime}(0)=-1+e^{-y}+y>0$, it is clear that the extremum is a maximum. So, we have $g(0)=0$, and for $0<y \leqslant 1, g(x)$ is monotonically increasing, whereas for $y>1$, it is monotonically increasing up to $\bar{x}$ and then monotonically decreasing to $e^{-y}>0$. Hence $g(x)>0$ for $x>0$, and we have proven the theorem for $n=2$.

Now, assuming it is true for $n-1$, we show that it holds for $n$.

For the general function $g_{n}(x):=f\left(x, x_{2}, \ldots, x_{n}\right)$ with fixed $x_{i} \geqslant 0$ for $i>1$, we note that $g_{n}(0)=0$ and

$$
\lim _{x \rightarrow \infty}=1-\prod_{i=2}^{n}\left(1-e^{-x_{i}}\right) \geqslant 0 .
$$

With $Q:=\prod_{i=2}^{n} x_{i}$, we get

$$
g_{n}^{\prime}(x)=e^{-x}\left(-\prod_{i=2}^{n}\left(1-e^{-x_{i}}\right)+Q e^{x(1-Q)}\right) .
$$

Evaluation at $x=0$ yields

$$
\begin{aligned}
g^{\prime}(0) & =Q-\prod_{i=2}^{n}\left(1-e^{-x_{i}}\right) \\
& \geqslant 1-e^{-Q}-\prod_{i=2}^{n}\left(1-e^{-x_{i}}\right) \\
& =f_{n-1}\left(x_{2}, x_{3}, \ldots, x_{n}\right) \\
& \geqslant 0
\end{aligned}
$$

where we have made use of the induction. Again, equality holds for $Q=0$ only. Solving $g_{n}^{\prime}(\bar{x})=0$ yields the single extremum

$$
\bar{x}=\frac{1}{Q-1} \ln \left(\frac{Q}{\prod_{i=2}^{n}\left(1-e^{-x_{i}}\right)}\right) .
$$

We already established in (36) that the numerator is greater than (or equal to) the denominator in logarithm, so, analogously to (33), we find that $\bar{x}<0$ for $0<Q<1$ and $\bar{x}>0$ for $Q>1$. Again, no solution for $Q=1$ and $g^{\prime}(x) \equiv 0$ for $Q=0$. This completes the proof. 


\section{Acknowledgments}

The author would like to thank Pascal Vontobel and Joachim Rosenthal for helpful discussions on the proof in the appendix. The partial support of the DARPA/IXO-NEST Program (AF-F30602-01-2-0526), NSF (ECS0225265), and ORAU is gratefully acknowledged.

\section{References}

1. G. J. Pottie and W. J. Kaiser, "Wireless integrated network sensors," Communications of the ACM, vol. 43, no. 5, pp. 551-558, 2000.

2. I. F. Akyildiz, W. Su, Y. Sankarasubramaniam, and E. Cayirci, "Wireless sensor networks: a survey," Computer Networks, vol. 38, pp. 393-422, Mar. 2002.

3. V. Rodoplu and T. H. Meng, "Minimum Energy Mobile Wireless Networks," IEEE Journal on Selected Areas in Communications, vol. 17, no. 8, pp. 1333-1344, 1999.

4. A. J. Goldsmith and S. B. Wicker, "Design Challenges for Energy-Constrained Ad Hoc Wireless Networks," IEEE Wireless Communications, vol. 9, pp. 8-27, Aug. 2002.

5. A. Ephremides, "Energy Concerns in Wireless Networks," IEEE Wireless Communications, vol. 9, pp. 48-59, Aug. 2002.

6. J. Laneman, D. Tse, and G. Wornell, "Cooperative Diversity in Wireless Networks: Efficient Protocols and Outage Behavior," IEEE Transactions on Information Theory. Submitted for publication. Available at: http://www.nd.edu/ jnl/pubs/it2002.pdf.

7. T. S. Rappaport, Wireless Communications - Principles and Practice. Prentice Hall, 1996. ISBN 0-13-375536-3.

8. T. J. Shepard, "A Channel Access Scheme for Large Dense Packet Radio Networks," in ACM SIGCOMM, (Stanford, CA), Aug. 1996. Available at: http://www . acm.org/sigcomm/sigcomm96/papers/shepard.ps.

9. M. Haenggi, "Probabilistic Analysis of a Simple MAC Scheme for Ad Hoc Wireless Networks," in IEEE CAS Workshop on Wireless Communications and Networking, (Pasadena, CA), Sept. 2002.

10. J. Laneman and G. Wornell, "Energy-Efficient Antenna Sharing and Relaying for Wireless Networks," in IEEE Wireless Comm. and Netw. Conf. (WCNC), (Chicago, IL), pp. 7-12, 2000.

11. J. E. Wieselthier, G. D. Nguyen, and A. Ephremides, "On the Construction of Energy-Efficient Broadcast and Multicast Trees in Wireless Networks," in IEEE INFOCOM, (Tel Aviv, Israel), pp. 585-594, Mar. 2000.

12. P. Gupta and P. R. Kumar, "Towards an Information Theory of Large Networks: An Achievable Rate Region," in IEEE International Symposium on Information Theory, (Washington DC), p. 159, 2001. 